Original Paper

\title{
Roles of Exosome-Like Vesicles Released from Inflammatory C2C12 Myotubes: Regulation of Myocyte Differentiation and Myokine Expression
}

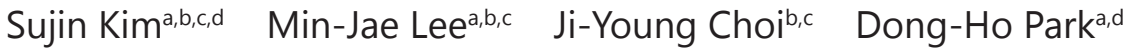 \\ Hyo-Bum Kwak ${ }^{a, d}$ Sohee Moon ${ }^{b, c}$ Je-Woo Koh ${ }^{b, c}$ Hun-Kyu Shin ${ }^{e}$ Ji-Kan Ryuc, ${ }^{c, f}$ \\ Chang-Shin Park ${ }^{b, c}$ Jai Hyung Parke Ju-Hee Kang ${ }^{b, c, d}$ \\ aDepartment of Kinesiology, Inha University, Incheon, bepartment of Pharmacology and Medicinal \\ Toxicology Research Center, Inha University, Incheon, 'Hypoxia-related Disease Research Center, Inha \\ University School of Medicine, Incheon, dWCSL, Inha University, Incheon, eDepartment of Orthopaedic \\ Surgery, Kangbuk Samsung Hospital, Sungkyunkwan University School of Medicine, Seoul, fDepartment \\ of Urology, Inha University School of Medicine, Incheon, Republic of Korea
}

\section{Key Words}

Skeletal muscle Inflammation Exosome - Myokine $\cdot$ Myogenic differentiation $\cdot$ Atrophy

\begin{abstract}
Background/Aims: The complicated differentiation processes of cells in skeletal muscle against inflammation that induce muscle atrophy are not fully elucidated. Given that skeletal muscle is a secretory organ, we evaluated the effects of inflammation on myogenic signals and myokine expression, and the roles of inflammatory exosomes released by myotubes in myogenic differentiation. Methods: Inflammation was induced by treatment of fully differentiated $\mathrm{C} 2 \mathrm{C} 12$ myotubes with a cytokine mixture of TNF- $\alpha$ and INF- $\gamma$. Exosome-like vesicles (ELVs) were isolated from conditioned media of control or inflamed myotubes and incubated with myoblasts. The expression of molecular switches that contribute to myogenic differentiation, including several kinases, their downstream targets, and myokines, were evaluated using immunoblot analysis in inflamed myotubes and in myoblasts treated with ELVs. Results: Inflammation activated molecular mechanisms contributing to muscle atrophy, including AMPK, p-38 MAPK and JNK, while inhibiting Akt-mediated myogenic signals. In addition, inflammation induced myostatin expression with suppression of a myostatincounteracting myokine, decorin. Well-characterized ELVs released from inflamed myotubes induced myoblast inflammation and inhibited myogenic mechanisms while stimulating atrophic signals. Conclusion: Inflammation of skeletal muscle induces muscle atrophy via
\end{abstract}

Dr. Ju-Hee Kang

And Dr. Jai Hyung Park
Department of Pharmacology and Medicinal Toxicology Research Center, Hypoxia-related Diseases Research Center, Inha University School of Medicine, Inha-ro, Nam-gu, Incheon 22212 (Korea) Tel. +82-32-860-9872, +82-2-2001-2473, E-mail johykang@inha.ac.kr; wonnypia@hanmail.net 


\section{Cellular Physiology Cell Physiol Biochem 2018;48:1829-1842

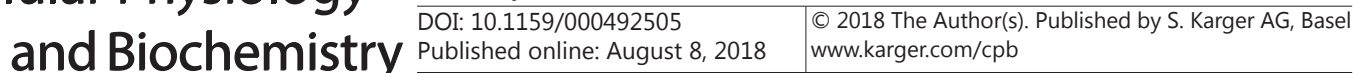 \\ Kim et al.: Effects of Exosomes on Muscle Differentiation in Inflamed Myotubes}

multiple mechanisms, including the regulation of myokines and kinases. Inflammatory ELVs are likely to contribute to inflammation-induced muscle atrophy.

\section{Introduction}

Inflammation in insulin-sensitive organs, including white adipose tissue (WAT), skeletal muscle, and liver, plays key roles in the development of chronic metabolic diseases. WAT is a source of obesity-induced chronic inflammation [1]; however, it is not the only organ that develops macrophage accumulation in obesity. In fact, recent studies have challenged the causal role of WAT inflammation in obesity-linked metabolic dysregulation of the whole body $[2,3]$. In addition, it was suggested that local inflammation and ectopic lipid accumulation in skeletal muscle leads to impaired insulin signaling [2]. Furthermore, inflammation in skeletal muscle and the accumulation of macrophages are observed not only in metabolic diseases but also in certain conditions, such as aged muscle [4], acute muscle damage by high-intensity eccentric exercise $[5,6]$, or chronic diseases (including cancer cachexia) [7], which leads to physical inactivity and aggravates the loss of skeletal muscle oxidative capacity, and vice versa [8]. In contrast, regular exercise with optimal intensity decreases the levels of inflammation in skeletal muscle and mitigates aging-induced muscle atrophy [2, 9]. Skeletal muscle is the largest organ of the body in nonobese individuals and is important in the regulation of whole-body metabolism. Skeletal muscle cells dynamically respond to physiological and/or pathological stimuli, including inflammation, leading to either skeletal muscle atrophy or hypertrophy. Myogenic differentiation consists of complicated events that determine the cell cycle, the characteristic pattern of skeletal muscle gene expression, and myotube formation. The commitment of myogenic satellite cells to myotube formation is regulated by multiple kinases, including mitogen-activated protein kinases (MAPKs), 5'-AMPactivated protein kinase (AMPK), mammalian target of rapamycin (mTOR), and PI3K/Akt $[10-14]$, and by muscle regulatory transcription factors, including MyoD and myogenin [15, 16].

During the past decade, several lines of evidence have shown that skeletal muscle is also a secretory organ: the secretion of muscle-cell-derived effector molecules, "myokines", may influence metabolism in tissues through intercellular communication with an endocrine effect [17]. Moreover, probably in an autocrine or paracrine fashion, myokines may regulate skeletal muscle metabolism and differentiation. Using proteomics approaches, several myokines have been identified in human myotubes [18-20]. Importantly, various myokines were found in secretory intercellular communicating vesicles, particularly in exosomes [21, 22]. In addition, myokines are closely associated with muscle atrophy and with the regulation of inflammation in skeletal muscle [23, 24]. However, the role of inflammation in the expression of myokines in skeletal muscle cells has not been fully elucidated. Furthermore, the effects of inflammatory exosomes on the myogenic differentiation of myoblasts have not been evaluated. Therefore, we hypothesized that inflammation alters myokine expression in skeletal muscle cells and that exosomes released from inflammatory muscle cells are responsible for suppressing muscle differentiation. Among myokines, in particular, it has been reported that decorin downregulates the activity of the anti-myogenic myostatin/Smad [25-27] and is associated with muscle regeneration [10], hypertrophy [28], and myogenic differentiation [29]. Therefore, we evaluated the effects of inflammation on the expression of decorin and myostatin and on the molecular switches of muscle differentiation using C2C12 myotubes. 


\section{Cellular Physiology Cell Physiol Biochem 2018;48:1829-1842 \begin{tabular}{l|l} 
and Biochemistry Published online: August 8, 2018 & $\begin{array}{l}\text { C } 2018 \text { The Author(s). Published by S. Karger AG, Basel } \\
\text { www.karger.com/cpb }\end{array}$
\end{tabular} Kim et al.: Effects of Exosomes on Muscle Differentiation in Inflamed Myotubes}

\section{Materials and Methods}

\section{Cell culture and treatment}

Murine myoblasts (C2C12) were obtained from the American Type Culture Collection (Manassas, VA, USA) and grown in Dulbecco's Modified Eagle's Medium (DMEM, Invitrogen, Carlsbad, CA, USA) supplemented with 10\% fetal bovine serum (FBS, HyClone, GE Healthcare Bio-Sciences, Pittsburgh, PA, USA) and antibiotics (100 U/mL penicillin and $100 \mu \mathrm{g} / \mathrm{mL}$ streptomycin) in humid air containing $5 \% \mathrm{CO}_{2}$ at $37^{\circ} \mathrm{C}$. Monolayers of undifferentiated $\mathrm{C} 2 \mathrm{C} 12$ myoblasts were kept in a proliferative state in DMEM with $10 \%$ FBS, and myoblasts at $\sim 100 \%$ confluency (day 0 ) were differentiated into myotubes by switching the proliferation medium to differentiation medium (DMEM supplemented with $2 \%$ horse serum), as described previously [30]. The differentiation medium was changed daily until day 5 when myotubes were clearly observed. We used serum-free DMEM containing a cytokine mixture of interferon- $\gamma$ (IFN- $\gamma$; R\&D Systems, Minneapolis, MN, USA) and tumor necrosis factor- $\alpha$ (TNF- $\alpha$; R\&D Systems) to induce the inflammatory responses of myotubes [31]). Inflammatory exosome-like vesicles (ELVs) were enriched from the conditioned medium (CM) of myotubes that were treated with the cytokine mixture or vehicle for $24 \mathrm{~h}$. Undifferentiated myoblasts were treated with serum-free DMEM with ELVs enriched from inflammatory CM or control CM for the indicated time (see below). For ELV treatment, culture media of C2C12 myoblasts at $\sim 100 \%$ confluency were replaced every $24 \mathrm{~h}$ by serum-free media containing ELVs with $10 \mu \mathrm{g}$ of exosomal proteins.

\section{Enrichment of exosome-like vesicles}

To enrich for ELVs, C2C12 myoblasts were plated on $100 \mathrm{~mm}$ dishes at a density of $1 \times 10^{6}$ cells and differentiated for 6 days as described above. After washing the C2C12 myotubes with serum-free DMEM, to clear exogenous ELVs in FBS, cells were treated with the cytokine mixture or phosphate-buffered saline (PBS) for $24 \mathrm{~h}$ in serum-free medium. We enriched ELVs by differential centrifugation of CM combined with microfiltration, as described previously [32]. Briefly, CM either from control or inflamed myotubes were collected from six $100 \mathrm{~mm}$ dishes per group and were centrifuged at $300 \mathrm{x} g$ for $10 \mathrm{~min}$ at room temperature (RT), to discard dead cells and bulky cellular debris. Subsequently, the supernatants were centrifuged at $2000 \mathrm{x}$ for $10 \mathrm{~min}$ at $4^{\circ} \mathrm{C}$. To remove contaminating apoptotic bodies, microvesicles, and small cell debris, supernatants were filtered through a Millex syringe filter fitted with a $0.22 \mu \mathrm{m}$ membrane (Merck Millipore, Temecula, CA, USA). The filtrate was then subjected to $100,000 \mathrm{x} g$ centrifugation in a Type70 Ti rotor for 90 min at $4{ }^{\circ} \mathrm{C}$, to pellet ELVs. The supernatant was carefully removed, and pellets were washed with PBS by $100,000 \mathrm{x} g$ centrifugation for $70 \mathrm{~min}$, resuspended in $200 \mu \mathrm{L}$ of PBS and stored at $-80^{\circ} \mathrm{C}$ until use. The purity of ELVs was determined by detecting the presence of an exosomal marker (Alix) and the absence of a nonexosomal marker (golgin subfamily A member 2; GM130) by immunoblot analysis.

\section{Western blot}

To prepare whole-cell lysates, cells were washed twice with ice-cold PBS, scraped into ice-cold radioimmunoprecipitation (RIPA) buffer (Sigma-Aldrich) containing protease inhibitors and phosphatase inhibitors (Sigma-Aldrich), and sonicated to homogenize the cell suspension. The cell debris of the homogenates was removed by centrifugation at $13,000 \times \mathrm{g}$ for $10 \mathrm{~min}$ and total protein content were quantified using a BCA protein assay kit (Pierce, Rockford, IL, USA). Aliquots of protein extracts (10-30 $\mu \mathrm{g}$ ) were separated by SDS-polyacrylamide gel electrophoresis (SDS-PAGE) and transferred to a nitrocellulose membrane in transfer buffer containing $25 \mathrm{mM}$ Tris- $\mathrm{HCl}, 192 \mathrm{mM}$ glycine, and $20 \%$ methanol. The membrane was blocked with $5 \%$ bovine serum albumin or non-fat milk in Tris-buffered saline with $0.2 \%$ Tween 20 and incubated with specific primary antibodies (Table 1). After washing, membranes were incubated for $1 \mathrm{~h}$ at RT with horseradish-peroxidase-conjugated secondary antibodies. Immunoreactive bands were visualized using an enhanced chemiluminescence detection system (Pierce) and analyzed using image analysis software Quantity One ${ }^{\circledast}$ 4.6. The protein content in isolated ELVs was quantified by a BCA protein quantitation kit after 1:1 dilution with RIPA buffer, and myoblasts were treated with exosomes containing $10 \mu \mathrm{g}$ of protein per $60-\mathrm{mm}$ dish $(3.3 \mathrm{microg} / \mathrm{mL})$, as indicated. Ten micrograms of exosomal proteins were mixed with reducing sample buffer (50 mM Tris- $\mathrm{HCl}$ (pH 6.8), $10 \%$ glycerol, $2 \% \mathrm{SDS}, 100 \mathrm{mM}$ dithiothreitol,

\section{KARGER}




\section{Cellular Physiology Cell Physiol Biochem 2018;48:1829-1842 \begin{tabular}{l|l|l} 
DOI: 10.1159/000492505 & O 2018 The Author(s). Published by S. Karger AG, Basel \\
and Biochemistry & $\begin{array}{l}\text { Published online: August 8, } 2018 \\
\text { www.com/cpb }\end{array}$
\end{tabular} \\ Kim et al.: Effects of Exosomes on Muscle Differentiation in Inflamed Myotubes}

and $0.01 \%$ bromophenol blue), and proteins were resolved by SDS-PAGE, transferred to a nitrocellulose membrane, blocked with $5 \%$ non-fat milk in TBS-T ( $0.1 \%$ Tween 20$)$, and probed with an anti-Alix antibody or an anti-GM130 antibody.

\section{Characterization of ELVs by electron microscopy and dynamic light scattering analysis}

ELVs were visualized using transmission electron microscopy (TEM) according to Lobb, et al. [32], with slight modifications. Briefly, two microliters of the fresh ELV solution without fixation was transferred onto Formvar-carbon-coated EM grids and allowed to dry at RT. Grids were then stained with $3 \%$ uranyl acetate for $10 \mathrm{~min}$. Imaging was performed at an acceleration voltage of $75 \mathrm{kV}$ using a CCD camera (5 x 5 k, Hamamatsu Photonics, Shizuoka, Japan) coupled to an H-7100 electron microscope (Hitachi High Technology, Tokyo, Japan).

The size distribution of ELVs was determined by dynamic light scattering (DLS) analysis. Aliquots of ELV suspensions containing $100 \mu \mathrm{g}$ of protein were diluted in PBS to a total volume of $1 \mathrm{~mL}$ and $3 \times 10$ measurement runs were performed, with standard settings (Refractive Index $=1.331$, viscosity $=0.89$, temperature $=25^{\circ} \mathrm{C}$ ). The shape of the particle size distribution and the intensity peak were measured to determine the agreement of ELVs with the known size distribution of exosomes (30-150 $\mathrm{nm})$.

\section{Statistics}

All data are presented as means \pm S.D. The effects of inflammation or inflammatory ELVs on the expression of proteins were compared via the Mann-Whitney $U$ test or one-sample t-test,

Table 1. Antibodies used in this study

\begin{tabular}{|c|c|c|c|c|}
\hline Antibodies & Source & Dilution & Manufacturer & Catalog\# \\
\hline anti-AMPK & Rabbit & 1:1000 & Cell Signaling & $\# 2532$ \\
\hline anti-p-AMPK Thr172 & Rabbit & $1: 1000$ & Cell Signaling & $\# 2535$ \\
\hline anti-ACC & Rabbit & $1: 1000$ & Cell Signaling & $\# 3662$ \\
\hline anti-p-ACCser79 & Rabbit & 1:1000 & Cell Signaling & $\# 3661$ \\
\hline anti-iNOS & Rabbit & 1:1000 & Santa Cruz Biotechnology & Sc-651 \\
\hline anti-COX-2 & Rabbit & $1: 1000$ & Abcam & ab15191 \\
\hline anti-raptor & Rabbit & 1:1000 & Cell Signaling & $\# 2280$ \\
\hline anti-p-raptor ser792 & Rabbit & 1:1000 & Cell Signaling & $\# 2083$ \\
\hline anti-mTOR & Rabbit & 1:1000 & Cell Signaling & $\# 2972$ \\
\hline anti-p-mTOR $\operatorname{ser2448}$ & Rabbit & 1:1000 & Cell Signaling & $\# 2971$ \\
\hline anti-Akt & Rabbit & $1: 1000$ & Cell Signaling & $\# 9272$ \\
\hline anti-p-Aktser473 & Rabbit & $1: 1000$ & Cell Signaling & $\# 9271$ \\
\hline anti-P38MAPK & Mouse & $1: 1000$ & Cell Signaling & $\# 9217$ \\
\hline anti-p-P38MAPK Thr $180 / 1$ Tyrr182 & Rabbit & $1: 1000$ & Cell Signaling & $\# 9215$ \\
\hline anti- SAPK/JNK & Rabbit & $1: 1000$ & Cell Signaling & $\# 9252$ \\
\hline anti-p- SAPK/JNKThr 183/tyrr185 & Rabbit & 1:1000 & Cell Signaling & $\# 9251$ \\
\hline anti-FOXO3a & Rabbit & $1: 1000$ & Cell Signaling & $\# 2497$ \\
\hline anti-p-FoxO1 tura//FoxO3a Tur32 & Rabbit & 1:1000 & Cell Signaling & $\# 9464$ \\
\hline anti-P70S6K & Rabbit & $1: 1000$ & Cell Signaling & $\# 9202$ \\
\hline anti-p-P70S6K Thrss9 & Mouse & 1:1000 & Cell Signaling & $\# 9206$ \\
\hline anti-atrogin1 & Mouse & 1:1000 & Santa Cruz Biotechnology & sc-166806 \\
\hline anti-myo-D & Rabbit & $1: 500$ & Santa Cruz Biotechnology & sc-304 \\
\hline anti-myogenin & Mouse & $1: 500$ & Santa Cruz Biotechnology & sc- -12732 \\
\hline anti-decorin & Rabbit & 1:500 & Santa Cruz Biotechnology & sc-22753 \\
\hline anti-myostatin & Rabbit & $1: 500$ & Abcam & ab-71808 \\
\hline anti-Alix & Mouse & $1: 1000$ & Cell Signaling & 2171 \\
\hline anti-GM130 & Mouse & 1:1000 & BD Transduction & 610822 \\
\hline anti- $\alpha$-actinin & Rabbit & 1:1000 & Santa Cruz Biotechnology & sc-166524 \\
\hline anti- $\beta$-actin & Rabbit & $1: 10000$ & Prosci & PM-7547 \\
\hline
\end{tabular}

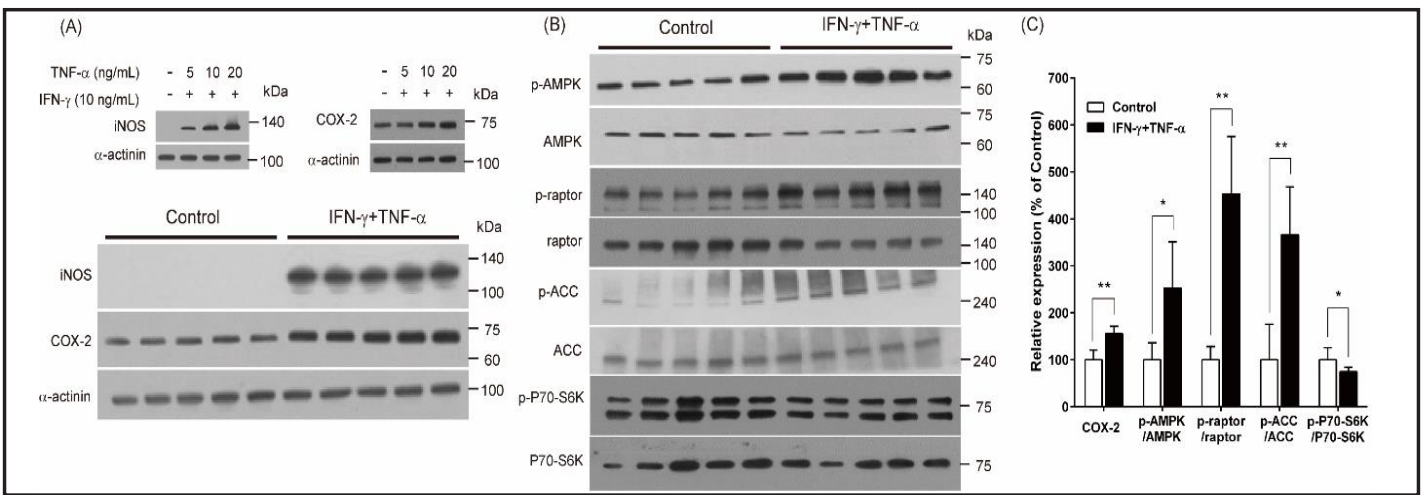

Fig. 1. Induction of cytokine-mediated inflammation with concurrent activation of AMPK signaling pathways in C2C12 myotubes. (A) Inflammation of C2C12 myotubes by treatment with a TNF- $\alpha$ and INF- $\gamma$ mixture was evaluated by the induction of iNOS and COX-2 expression, which was dependent on the dose of TNF- $\alpha$. (B, C) Treatment of myotubes with a cytokine mixture of IFN- $\gamma(10 \mathrm{ng} / \mathrm{mL})$ and TNF- $\alpha$ (20 ng/ $\mathrm{mL}$ ) significantly increased the level of p-AMPK and of its downstream effectors, p-ACC and p-raptor, while it significantly decreased the level of phosphorylated P70-S6K. Quantitation of the band densities showed statistical significance based on the Mann-Whitney U test. ${ }^{*} \mathrm{p}<0.05$; ${ }^{* *} \mathrm{p}<0.01$ vs the control. Vertical bars indicate mean levels of protein expression relative to control group that were normalized to themselves to be $100 \%$ of mean value with S.D. regarding the variability in replicates. 


\section{Cellular Physiology Cell Physiol Biochem 2018;48:1829-1842

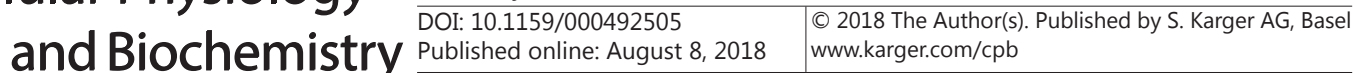 \\ Kim et al.: Effects of Exosomes on Muscle Differentiation in Inflamed Myotubes}

respectively, using the GraphPad Prism program version 6.0 (GraphPad Software, San Diego, CA, USA). We considered $P<0.05$ as significant.

\section{Results}

Induction of inflammation activates AMPK signaling pathways in myotubes As expected, when we treated C2C12 myotubes with a cytokine mixture of IFN- $\gamma(10 \mathrm{ng} / \mathrm{mL})$ and TNF- $\alpha$ (5-20 ng/ $\mathrm{mL}$ ) for $24 \mathrm{~h}$, the expression of both inducible nitric oxide synthase (iNOS) and cyclooxygenase 2 (COX-2) was dramatically increased compared with cells without treatment, indicating that the cytokine mixture successfully induced myotube inflammation (Fig. 1A). A slight induction of inflammatory proteins (iNOS and COX-2) at $4 \mathrm{~h}$ after treatment of cells with a cytokine mixture (TNF- $\alpha 20 \mathrm{ng} /$ $\mathrm{mL}+\mathrm{IFN}-\gamma 10 \mathrm{ng} / \mathrm{mL}$ ) was also observed, but the induction was not apparent (data not shown); therefore, we selected 24 $\mathrm{h}$ as the time point of inflammation for further experiments. Given that AMPK is an important regulator of pathways associated with intracellular metabolism and inflammatory responses [33], we evaluated the effects of inflammation on AMPK activity and on its downstream molecules of mTORC1 [34]. Consistent with a previous study [35], the induction of inflammation in differentiated C2C12 myotubes significantly increased p-AMPK at Thr172 and its downstream molecules p-ACC at Ser79 and p-raptor at Ser792, which was accompanied by a decreased phosphorylation of P70-S6K at Thr421/Ser424 (Fig. 1B, C). The AMPK-mTOR pathway is also associated with autophagy, hence we tested whether autophgy signals are activated in myotubes treated with serum-free complete DMEM as compared with myotubes without serum starvation. However, we couldn't detect a significant activation of autophagy by serum starvation (Fig. 2), indicating that the effects of cytokines in serum-free media on AMPK pathway is likely to be induced by inflammation, not by activation of autophagy.

\section{Effects of inflammation on myoblast differentiation and myokine expression}

To evaluate the effect of inflammation on the molecules that are involved in muscle atrophy, we measured the expression of MAFbx (also known as atrogin-1 or F-box only protein 32), a key molecule in skeletal muscle atrophy [36] in inflamed myotubes. As shown in Fig. 3A, treatment of differentiated C2C12 with a cytokine mixture for $24 \mathrm{~h}$ increased the expression of MAFbx in a TNF- $\alpha$ dose-dependent manner $(5-20 \mathrm{ng} / \mathrm{mL}$ ), concurrent with iNOS induction. Conversely, the level of MyoD protein, a marker of myogenic differentiation, in myotubes treated with a cytokine mixture was significantly lower than that observed in vehicle-treated myotubes. Another marker of myogenic differentiation, myogenin, was slightly downregulated by inflammatory stimuli. In addition, the expression of decorin, a myokine with myogenic activity [10, 29], was suppressed in a dose-dependent manner (Fig. $3 \mathrm{~A})$. 


\section{Cellular Physiology Cell Physiol Biochem 2018;48:1829-1842 \begin{tabular}{l|l} 
and Biochemistry Published online: August 8, 2018 & $\begin{array}{l}\text { D } 2018 \text { The Author(s). Published by S. Karger AG, Basel } \\
\text { www.karger.com/cpb }\end{array}$
\end{tabular}
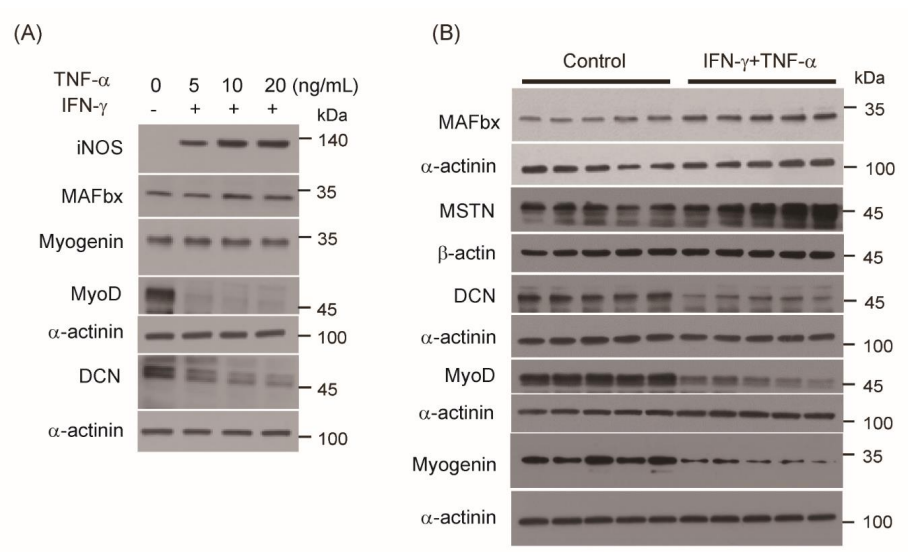

(C)

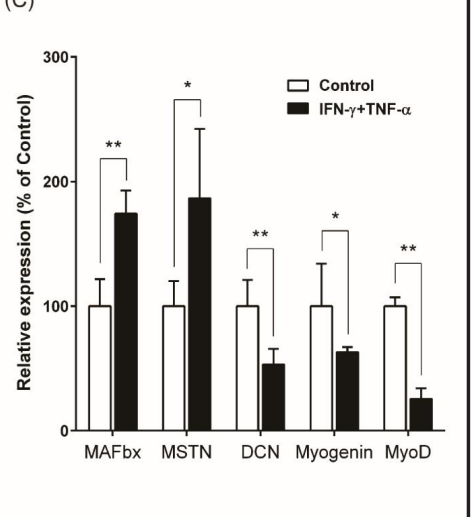

Fig. 3. Inflammation of myotubes suppresses myogenic signals but increases the levels of proteins responsible for muscle atrophy. (A) Treatment of myotubes with a cytokine mixture remarkably suppressed the expression of Myo-D and decorin (DCN) but increased the level of MAFbx. A representative blot from a duplicate experiment is presented. $(B, C)$ Induction of inflammation significantly increased the levels of myostatin (MSTN) and MAFbx, but significantly decreased the levels of decorin (DCN), MyoD, and myogenin. Quantitation of the band densities showed statistical significance based on the Mann-Whitney U test. ${ }^{*} \mathrm{p}<0.05 ;{ }^{* *} \mathrm{p}<0.01$ vs the control. Vertical bars indicate mean levels of protein expression relative to control group that were normalized to themselves to be $100 \%$ of mean value with S.D. regarding the variability in replicates.

Given that myostatin, another molecule that is involved in muscle atrophy, is counteracted by decorin $[25,27,37]$ and is closely associated with AMPK-mediated energy metabolism and atrophy [38-41], we evaluated the expression of myostatin in inflamed C2C12 myotubes. We found that the expression levels of myostatin and MAFbx in C2C12 myotubes was significantly increased, while the levels of decorin, MyoD, and myogenin were significantly decreased after treatment with a cytokine mixture for $24 \mathrm{~h}$ (Fig. 3B, C).

\section{Effects of inflammation on p38-MAPK, JNK, and Akt phosphorylation in myotubes}

Independently of the AMPK/mTOR signaling pathways, the activation of PI3K/Akt is crucial for muscle differentiation [42], while the c-jun NH2 terminus kinase (JNK) [43] and p38-MAPK [11] are anti-myogenic MAPKs. Therefore, we evaluated the effects of inflammation on these signaling pathways. As shown in Fig. 4, the levels of phosphorylated p38-MAPK and p-JNK were significantly higher in myotubes treated with a cytokine mixture than they were in the vehicle-treated control. In contrast, we observed a significant suppression of the expression of p-Akt at Ser473 and its downstream p-FOXO3a at Thr24/32 and p-mTOR at Ser2448 in inflamed myotubes compared with the controls (Fig. 4).

\section{Exosome-like vesicles released from inflamed myotubes inhibit expression of MyoD and myogenin}

We confirmed that the $100,000 \times g$ pellets were enriched with exosomes via the by detection of an exosomal marker and exclusion of a non-exosomal marker through immunoblotting, TEM imaging, and analysis of the size distribution of ELVs by DLS. The expression of Alix in the ELV fraction was clearly observed, and the level of Alix in ELVs from inflamed myotubes was higher than that detected in control ELVs; in addition, we did not detect GM130, a non-exosomal protein, in the two types of ELVs (but found it in cell lysate) (Fig. 5A). Furthermore, the size distribution of particles in the ELV suspension (z-ave $=145.9 \pm 25.6 \mathrm{~nm}$ ), with a peak intensity around $90 \mathrm{~nm}$, was compatible with the known size distribution of exosomes (Fig. 5B). A morphological analysis using TEM also revealed a heterogeneous population of vesicles with a heterogeneous diameter size ranging from 30 to $120 \mathrm{~nm}$ and round or cup-shaped particles (Fig. 5C). To evaluate the effects of ELVs from 


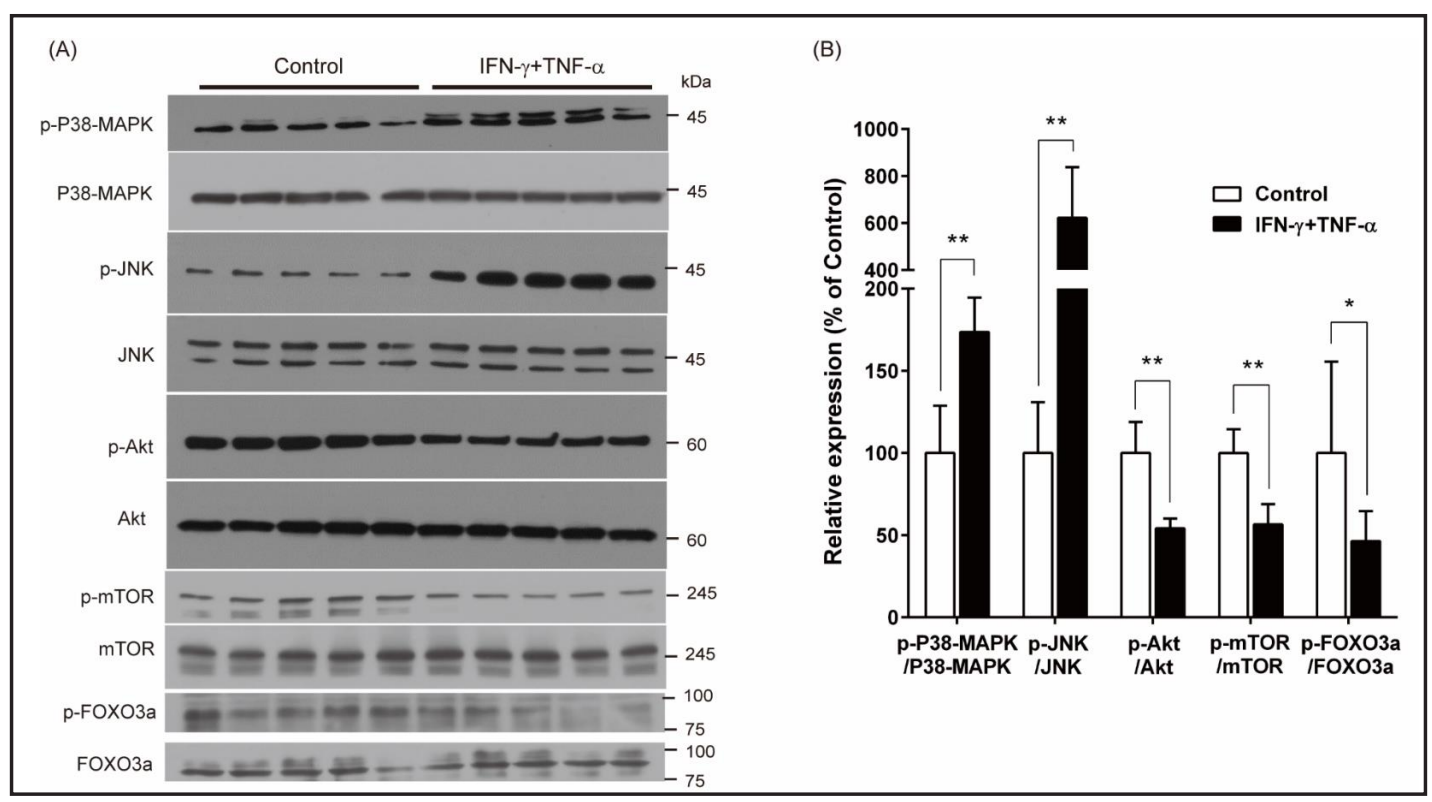

Fig. 4. Effects of inflammation on the phosphorylation of anti-myogenic MAPKs, and p-Akt-mediated phosphorylation of mTOR and FOXO3a. (A) Immunoblots of anti-myogenic MAPK phosphorylation and Akt-mediated myogenic signaling. Treatment of myotubes with a cytokine mixture dramatically increased MAPK activity, while it significantly inhibited Akt-mediated pathways. Inhibition of the Akt-mediated phosphorylation of FOXO3 by cytokines may cause MAFbx upregulation (Fig. 2B and 2C). (C) Quantitation of the band densities showed statistical significance based on the Mann-Whitney U test. ${ }^{*} \mathrm{p}<0.05 ;{ }^{* *} \mathrm{p}<0.01$ vs the control. Vertical bars indicate mean levels of protein expression relative to control group that were normalized to themselves to be $100 \%$ of mean value with S.D. regarding the variability in replicates.

Fig. 5. Characterization of exosome-like vesicles (ELVs) and the effects of ELVs released from myotubes on the levels of proteins associated with differentiation. myoblast

ELVs prepared from the conditioned medium of control myotubes (CEx) or inflamed myotubes (IEx) by differential c e n t r if u g a ti o n exhibited expression of an exosome marker (Alix) and absence of a non-exosomal marker (GM130). Ten micrograms of exosomal proteins were separated using SDS-PAGE followed by immunoblot, and a representative blot from duplicate experiments is shown. (B) Size distribution of ELVs measured by dynamic light scattering analysis. The size distribution showed a diameter that was within the range of that of exosomes (30-150 nm). We measured the size distribution of ELVs prepared from two independent experiments and obtained a similar result. (C) TEM imaging of ELVs with negative staining showed vesicles with a heterogeneous size of 30-120 nm (asterisks). A representative image from triplicate experiments is shown. (D) Effects of treatment with CEx- or IEx-containing serum-free DMEM on the level of the myogenic proteins myoD and myogenin. $\alpha$-Actinin was used as a loading control. A representative blot from duplicate experiments is shown. " $\mathrm{p}<0.05$ versus the levels in CEx-treated myoblasts $(n=3$, one-sample t-test). HS, myotubes fully differentiated by treatment with $2 \%$ horse serum for 5 days; SF, myoblasts treated with serum-free DMEM for 5 days.

\section{KARGER}


Fig. 6. Effects of exosomelike vesicles (ELVs) released from myotubes on the molecular pathways related to myogenesis. Effects of control or inflammatory exosomelike vesicles on (A, B) the activation of the AMPK and Akt pathways, which are associated with myoblast differentiation; (C) the level of cyclooxygenase 2 (COX2) and the phosphorylation of p38 MAPK and JNK; (D) the expression of myostatin (MSTN) and decorin (DCN); and (E) the FOXO3a-mediated MAFbx expression. $\alpha$-Actinin and $\beta$-actin were used as a loading control. Representative blots from triplicate experiments are presented. HS, myotubes differentiated by treatment with $2 \%$ horse serum for 5 days; SF, myoblasts treated with serum-free DMEM for 5 days; CEx, myoblasts treated with control ELVs; IEx, myoblasts treated with inflammatory ELVs. ${ }^{*} \mathrm{p}<0.05 ; \quad{ }^{* *} \mathrm{p}<0.01 \quad$ versus the levels in CEx-treated myoblasts $(n=3$, onesample t-test).

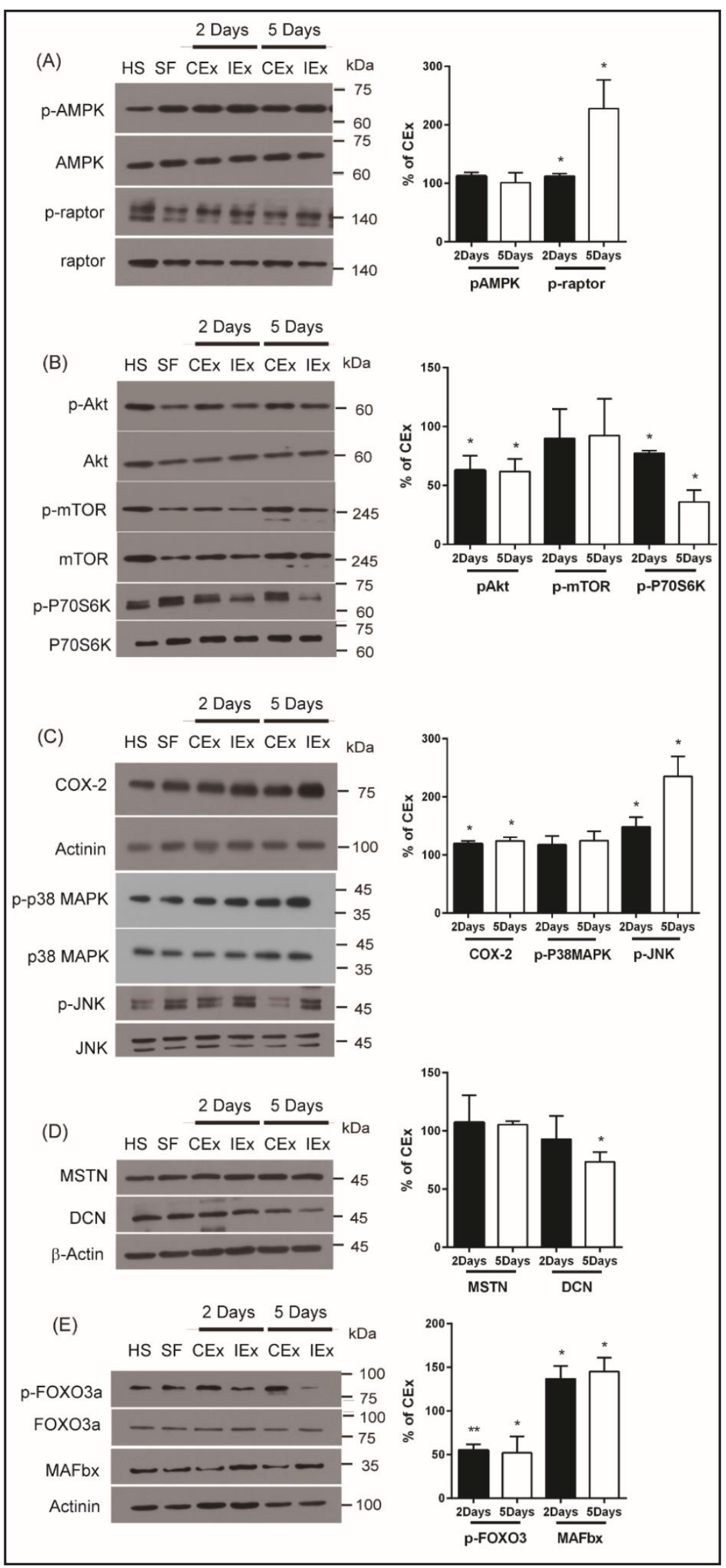




\section{Cellular Physiology Cell Physiol Biochem 2018;48:1829-1842

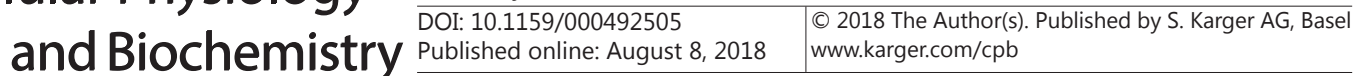 \\ Kim et al.: Effects of Exosomes on Muscle Differentiation in Inflamed Myotubes}

inflamed myotubes, we treated myoblasts grown in $3 \mathrm{~mL}$ of serum-free media with exosomes containing $10 \mu \mathrm{g}$ of proteins that were quantified by BCA assay. Interestingly, the levels of MyoD and myogenin in myoblasts treated with ELVs released from control myotubes (CEx) for 2 or 5 days were higher than those observed in cells treated with serum-free media for 5 days, indicating that CEx might induce myoblast differentiation [44]. The levels of MyoD and myogenin in myoblasts treated with CEx for 5 days were lower than those in 2-day-treated myoblasts, indicating that the relative effect size of serum starvation for different duration is larger than that of CEx treatment. Treatment of C2C12 myoblasts with ELVs released from inflamed myotubes (IEx) led to a decrease in the levels of both MyoD and myogenin compared with myoblasts treated with CEx or fully differentiated myotubes (Fig. 5D).

\section{Effects of exosome-like vesicles on signaling pathways associated with differentiation}

To test the anti-myogenic effects of inflammatory ELVs, we evaluated myogenic signals in ELV-treated myoblasts. When we treated myoblasts with CEx or IEx for 5 days, although p-AMPK levels were comparable, but p-raptor levels in IEx-treated myoblasts were 2.2-fold higher than those in CEx-treated cells (Fig. 6A). In contrast, p-Akt and p-P70S6K levels were significantly downregulated by IEx treatment compared with myoblasts treated with CEx, and p-mTOR levels in IEx-treated cells were slightly lower than CEx-treated cells (Fig. 6B). Compared with fully differentiated myotubes that were induced by $2 \%$ horse serum for 5 days, cells treated with CEx for 5 days showed slightly higher levels of p-AMPK, while the levels of phosphorylation of Akt and mTOR were comparable. The level of p-raptor in myoblasts treated with CEx for 2 days was comparable, while it was lower after 5 days than the level of fully differentiated myotubes. However, cells treated with IEx for 5 days showed lower levels of p-Akt and p-mTOR, but higher levels of p-AMPK compared with those observed in fully differentiated myotubes. We found that the levels of COX-2 in myoblasts treated with IEx for both 2 and 5 days were significantly higher than those detected in matched CEx-treated myoblasts or fully differentiated myoblasts. Considering the anti-myogenic MAPK pathways, myoblasts treated with IEx for 5 days showed the significantly higher level of p-JNK than that of CEx-treated myoblasts or fully differentiated myotubes (Fig. 6C). Furthermore, the expression level of decorin in myoblasts treated with IEx for 5 days was slightly but significantly lower than that detected in the matched CEx-treated myoblasts, but not in those treated for 2 days, while the levels of myostatin in IEx-treated myoblasts were not significantly different from those of CEx-treated cells (Fig. 6D). In addition, myoblasts treated with IEx for 5 days exhibited a reduced level of p-FOXO3a at Thr24/32, which is a target of Akt and causes upregulation of MAFbx [45], compared with CEx-treated myoblasts. Concurrently, the levels of MAFbx in IEx-treated myoblasts were significantly higher than those in CEx-treated cells (Fig. 6E).

\section{Discussion}

Inflammation of skeletal muscle has been implicated as a cause of the metabolic dysfunction and muscle atrophy that occur as a result of aging [46] and chronic diseases $[7,47]$, which are mediated by the elevation of pro-inflammatory cytokines. In contrast, exercise-induced, acute, localized, and transient inflammation promotes muscle repair and growth through, at least in part, the action of myokines [48]. In this study, we demonstrated that inflammation induced by treatment with a cytokine mixture turned on the molecular switches of muscle atrophy, but suppressed myogenic signaling. More importantly, ELVs from myotubes with inflammation is likely to contribute to the suppression of myogenic signaling pathways. In the face of stress or damages, an appropriate positive balance between the proliferation and differentiation of myogenic satellite cells in muscle against the loss of myocytes maintains the structure and function of skeletal muscles [49]. Our results are in agreement with previous studies $[50,51]$ in that inflammation can accelerate muscle atrophy in certain pathological conditions via mechanisms that inhibit both myogenic signals 


\section{Cellular Physiology Cell Physiol Biochem 2018;48:1829-1842

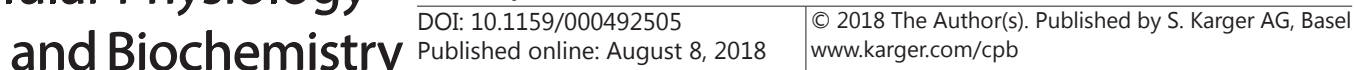 \\ Kim et al.: Effects of Exosomes on Muscle Differentiation in Inflamed Myotubes}

and energy-consuming protein synthesis. Recent studies provided evidence that myokines regulate myogenic differentiation and metabolism in skeletal muscle $[27,28,52]$. Consistently, our results clearly supported the contention that inflammation can lead to muscle atrophy, at least in part through the alteration of myokine expression; i.e., upregulation of myostatin associated with AMPK activation [41] and downregulation of counteracting decorin expression $[27,28]$. Therefore, although this cause-effect relationship should be clarified in further studies, the myogenic vs muscle atrophic effect of decorin and myostatin, respectively, may contribute to the inhibition of myogenesis by inflammation. Although pharmacological activation of AMPK is a strategy that has been used to reinforce anti-inflammatory mechanisms [53], AMPK activation can suppress muscle differentiation and contribute to muscle atrophy [40, 54]. Therefore, the consequences of activation of AMPK by muscular contraction or by cytokine-induced inflammation for muscle differentiation might be different. In addition to the AMPK- and myokineassociated inhibition of myogenesis, the cytokine-induced alteration of multiple myogenic signaling pathways observed in our model, including the activation of p38-MAPK [11] and JNK [43] and the inhibition of Akt [45], may contribute to the suppression of myogenesis by inflammation. The activation of AMPK and inhibition of Akt leads to the inhibition of mTOR-mediated myogenesis [13, $14]$, which may also negatively regulate myogenesis in inflamed muscle.

To the best of our knowledge, this is the first report that ELVs from inflamed myotubes significantly inhibited myogenic signals. Although we cannot exclude the possibility that the preparation of ELVs by differential ultracentrifugation of $\mathrm{CM}$ was contaminated with non-exosomal extracellular vesicles, the characterization of ELVs by DLS analysis, western blot analysis, and TEM imaging showed a high purity of these exosomes (Fig. 5). Exosomes contain numerousmoleculesthatare responsible for intercellular communication, including proteins, microRNAs, and lipids [55]. Decorin, but not myostatin, has been detected in human and mouse extracellular vesicles

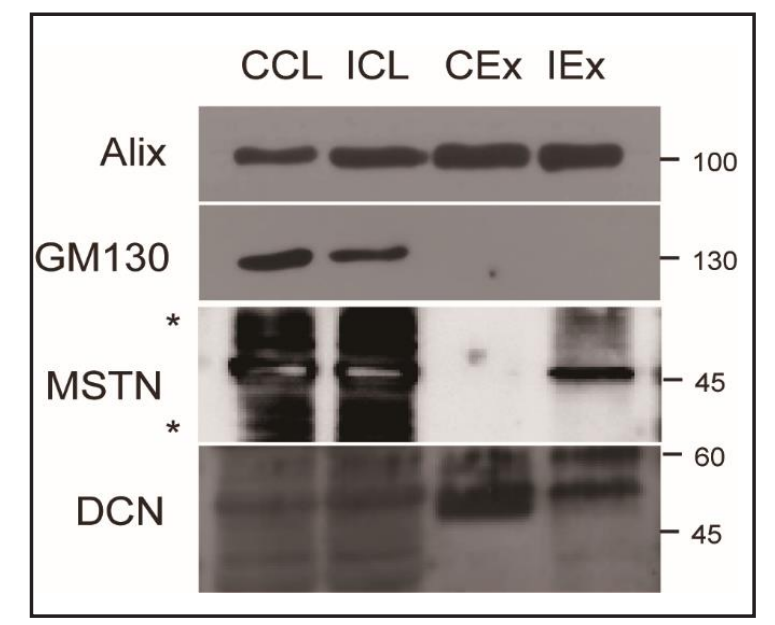

Fig. 7. Immunoblots of myostatin and decorin in exosome-like vesicles released from control or inflamed myotubes. The exosomal marker Alix was observed in cell lysates (CL) and exosome-like vesicles released from control myotubes (CEx) or inflamed myotubes (IEx), but non-exosomal protein GM130, a golgi matrix protein was not observed in exosomal fraction. In CEx, we observed the presence of decorin (DCN), but not myostatin (MSTN), however, both myokines were observed in IEx. Asterisks indicate nonspecific bands. CCL; control cell lysate, ICL; inflammatory cell lysate

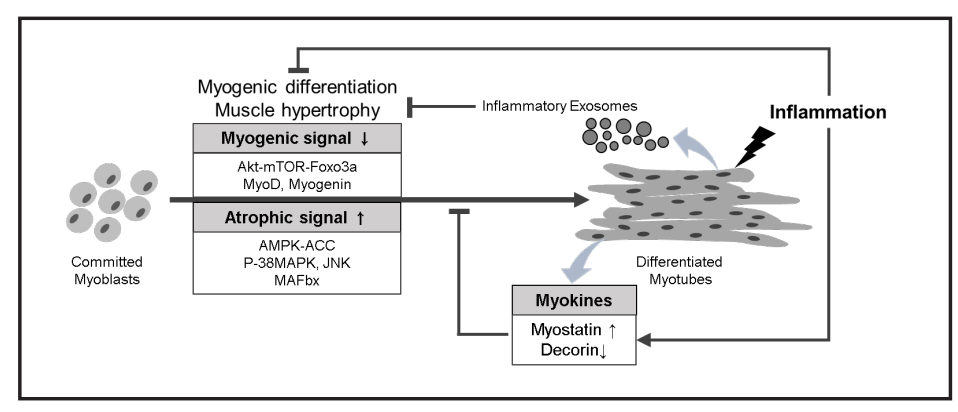

Fig. 8. Schematic summary of the mechanisms of inflammationinduced inhibition of myogenesis in $\mathrm{C} 2 \mathrm{C} 12$ cells. Inflammation induced by cytokine treatment inhibits various myogenic signals while it activates atrophic signals and alters the expression of myokines related to myogenesis. In addition, exosome-like vesicles released from inflamed myotubes contribute to anti-myogenesis. Through multiple mechanisms, inflammation inhibits myoblast differentiation to myotubes, which leads to muscle atrophy. 


\section{Cellular Physiology Cell Physiol Biochem 2018;48:1829-1842

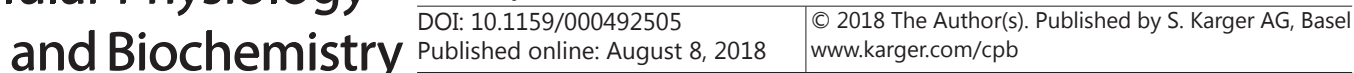 \\ Kim et al.: Effects of Exosomes on Muscle Differentiation in Inflamed Myotubes}

(www.exocarta.org and www.microvesicles.org). However, we detected myostatin in ELVs from inflammatory C2C12 myotubes, but not in control ELVs. The levels of decorin in both CEx and IEx were very low, but inflammation is likely to decrease the level of decorin in ELVs slightly (Fig. 7). Therefore, the possibility that the alteration of decorin and myostatin in ELVs contributes to the inhibition of myogenesis by inflammation in C2C12 could not be excluded. However, the biological roles of myostatin and decorin in inflammatory ELVs in myogenesis should be evaluated further, as we observed only a slight alteration in the levels of these myokines in myoblasts treated with inflammatory ELVs (Fig. 6). In addition, the possibility that unidentified proteins, miRNAs, or lipids in ELVs from inflammatory myotubes regulating myogenesis and myokine expression could not be ruled out [56-58]. Interestingly, our results suggested that ELVs released from myotubes can contribute to the activation or maintenance of muscle differentiation [44]; however, ELVs from inflammatory myotubes might hinder the process of differentiation (Figs 5D and 6). Although the molecular components that regulate myogenesis in ELVs released from myotubes should be further identified, our results provided, at least in part, evidence that inflammatory ELVs contribute to the inhibition of myoblast differentiation via multiple mechanisms, including disinhibition of the Akt-mediated suppression of FOXO3-MAFbx transcription, activation of the AMPK, p38-MAPK, and JNK signaling pathways, and induction of inflammation (Fig. 8).

In conclusion, our results clearly demonstrated that cytokine-induced inflammation significantly inhibited myogenic signaling and induced proteins responsible for muscle atrophy in a cellular model. The inflammation-induced inhibition of myogenic signals may be associated with an alteration in the levels of myokines and in the AMPK-, Akt-, and MAPKmediated pathways. Given the results that ELVs released from inflammatory myotubes inhibited myogenic signals, further characterization of the molecular components of ELVs is warranted for the elucidation of the mechanisms underlying inflammation-induced muscle atrophy.

\section{Acknowledgements}

The authors disclosed receipt of the following financial support for the research: This work was supported by Extramural grant of Hanmi Pharm. Co. Ltd. (to Jae-Hyung Park), WCSL at Inha University (to Ju-Hee Kang, Hyo-Bum Kwak and Dong-Ho Park), and Mid-career Research Program (2016R1A2B4008399 to Ju-Hee Kang) and Medical Research Center (2014009392 to Sujin Kim, So-Hee Moon, Je-Woo Koh, Ji-Young Choi, Chang-Shin Park, Ji-Kan Ryu and Ju-Hee Kang) through the National Research Foundation of Korea.

\section{Disclosure Statement}

No conflict of interests exists.

\section{References}

1 Weisberg SP, McCann D, Desai M, Rosenbaum M, Leibel RL, Ferrante AW, Jr.: Obesity is associated with macrophage accumulation in adipose tissue. J Clin Invest 2003;112:1796-1808.

-2 Jung DY, Ko HJ, Lichtman EI, Lee E, Lawton E, Ong H, Yu K, Azuma Y, Friedline RH, Lee KW, Kim JK: Shortterm weight loss attenuates local tissue inflammation and improves insulin sensitivity without affecting adipose inflammation in obese mice. Am J Physiol-Endoc M 2013;304:E964-976.

-3 Lee YS, Li P, Huh JY, Hwang IJ, Lu M, Kim JI, Ham M, Talukdar S, Chen A, Lu WJ, Bandyopadhyay GK, Schwendener R, Olefsky J, Kim JB: Inflammation is necessary for long-term but not short-term high-fat diet-induced insulin resistance. Diabetes 2011;60:2474-2483. 


\section{Cellular Physiology Cell Physiol Biochem 2018;48:1829-1842 \begin{tabular}{l|l} 
and Biochemistry & DOI: 10.1159/000492505 \\
Published 2018 The Author(s). Published by S. Karger AG, Basel \\
www.karger.com/cpb
\end{tabular}

4 Wang Y, Wehling-Henricks M, Samengo G, Tidball JG: Increases of M2a macrophages and fibrosis in aging muscle are influenced by bone marrow aging and negatively regulated by muscle-derived nitric oxide. Aging Cell 2015;14:678-688.

5 Child R, Brown S, Day S, Donnelly A, Roper H, Saxton J: Changes in indices of antioxidant status, lipid peroxidation and inflammation in human skeletal muscle after eccentric muscle actions. Clin Sci 1999;96:105-115.

6 Peake J, Nosaka K, Suzuki K: Characterization of inflammatory responses to eccentric exercise in humans. Exerc Immunol Rev 2005;11:64-85.

7 Bowen TS, Schuler G, Adams V: Skeletal muscle wasting in cachexia and sarcopenia: molecular pathophysiology and impact of exercise training. J Cachexia Sarcopeni 2015;6:197-207.

-8 Leermakers PA, Gosker HR: Skeletal muscle mitophagy in chronic disease: implications for muscle oxidative capacity? Curr Opin Clin Nutr Metab Care 2016;19:427-433.

-9 Petersen AM, Pedersen BK: The anti-inflammatory effect of exercise. J Appl Physiol 2005;98:1154-1162.

10 Li Y, Li J, Zhu J, Sun B, Branca M, Tang Y, Foster W, Xiao X, Huard J: Decorin gene transfer promotes muscle cell differentiation and muscle regeneration. Mol Ther 2007;15:1616-1622.

-11 Li YP, Chen Y, John J, Moylan J, Jin B, Mann DL, Reid MB: TNF-alpha acts via p38 MAPK to stimulate expression of the ubiquitin ligase atrogin1/MAFbx in skeletal muscle. FASEB J 2005;19:362-370.

12 Keren A, Tamir Y, Bengal E: The p38 MAPK signaling pathway: a major regulator of skeletal muscle development. Mol Cell Endocrinol 2006;252:224-230.

13 Goodman CA, Mayhew DL, Hornberger TA: Recent progress toward understanding the molecular mechanisms that regulate skeletal muscle mass. Cell Signal 2011;23:1896-1906.

14 Ge Y, Chen J: Mammalian target of rapamycin (mTOR) signaling network in skeletal myogenesis. J Biol Chem 2012;287:43928-43935.

15 Megeney LA, Kablar B, Garrett K, Anderson JE, Rudnicki MA: MyoD is required for myogenic stem cell function in adult skeletal muscle. Gene Dev 1996;10:1173-1183.

16 Karalaki M, Fili S, Philippou A, Koutsilieris M: Muscle regeneration: cellular and molecular events. In vivo 2009;23:779-796.

17 Pedersen BK, Febbraio MA: Muscles, exercise and obesity: skeletal muscle as a secretory organ. Nat Rev Endocrinol 2012;8:457-465.

18 Raschke S, Eckardt K, Bjorklund Holven K, Jensen J, Eckel J: Identification and validation of novel contraction-regulated myokines released from primary human skeletal muscle cells. PLoS One 2013;8:e62008.

19 Hartwig S, Raschke S, Knebel B, Scheler M, Irmler M, Passlack W, Muller S, Hanisch FG, Franz T, Li X, Dicken HD, Eckardt K, Beckers J, de Angelis MH, Weigert C, Haring HU, Al-Hasani H, Ouwens DM, Eckel J, Kotzka J, Lehr S: Secretome profiling of primary human skeletal muscle cells. Biochim Biophys Acta 2014;1844:1011-1017.

20 Norheim F, Raastad T, Thiede B, Rustan AC, Drevon CA, Haugen F: Proteomic identification of secreted proteins from human skeletal muscle cells and expression in response to strength training. Am J Physiol Endocrinol Metab 2011;301:E1013-1021.

-21 Safdar A, Saleem A, Tarnopolsky MA: The potential of endurance exercise-derived exosomes to treat metabolic diseases. Nat Rev Endocrinol 2016;12:504-517.

22 Lauritzen HP, Brandauer J, Schjerling P, Koh HJ, Treebak JT, Hirshman MF, Galbo H, Goodyear LJ: Contraction and AICAR stimulate IL-6 vesicle depletion from skeletal muscle fibers in vivo. Diabetes 2013;62:30813092.

23 Demontis F, Piccirillo R, Goldberg AL, Perrimon N: The influence of skeletal muscle on systemic aging and lifespan. Aging cell 2013;12:943-949.

24 Pedersen BK: Exercise-induced myokines and their role in chronic diseases. Brain Behav Immun 2011;25:811-816.

25 El Shafey N, Guesnon M, Simon F, Deprez E, Cosette J, Stockholm D, Scherman D, Bigey P, Kichler A: Inhibition of the myostatin/Smad signaling pathway by short decorin-derived peptides. Exp Cell Res 2016;341:187-195.

-26 Guiraud S, van Wittenberghe L, Georger C, Scherman D, Kichler A: Identification of decorin derived peptides with a zinc dependent anti-myostatin activity. Neuromuscul Disord 2012;22:1057-1068. 


\section{Cellular Physiology Cell Physiol Biochem 2018;48:1829-1842

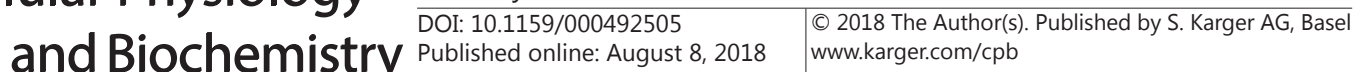

-27 Kishioka Y, Thomas M, Wakamatsu J, Hattori A, Sharma M, Kambadur R, Nishimura T: Decorin enhances the proliferation and differentiation of myogenic cells through suppressing myostatin activity. J Cell Physiol 2008;215:856-867.

28 Kanzleiter T, Rath M, Gorgens SW, Jensen J, Tangen DS, Kolnes AJ, Kolnes KJ, Lee S, Eckel J, Schurmann A, Eckardt K: The myokine decorin is regulated by contraction and involved in muscle hypertrophy. Biochem Biophys Res Commun 2014;450:1089-1094.

29 Geng J, Liu G, Peng F, Yang L, Cao J, Li Q, Chen F, Kong J, Pang R, Zhang C: Decorin promotes myogenic differentiation and mdx mice therapeutic effects after transplantation of rat adipose-derived stem cells. Cytotherapy 2012;14:877-886.

30 Jang EH, Moon JS, Ko JH, Ahn CW, Lee HH, Shin JK, Park CS, Kang JH: Novel black soy peptides with antiobesity effects: activation of leptin-like signaling and AMP-activated protein kinase. Int J Obesity 2008;32:1161-1170.

31 Kimura K, Cheng XW, Inoue A, Hu L, Koike T, Kuzuya M: beta-Hydroxy-beta-methylbutyrate facilitates PI3K/Akt-dependent mammalian target of rapamycin and Fox01/3a phosphorylations and alleviates tumor necrosis factor alpha/interferon gamma-induced MuRF-1 expression in C2C12 cells. Nutrition Res 2014;34:368-374.

-32 Lobb RJ, Becker M, Wen SW, Wong CS, Wiegmans AP, Leimgruber A, Moller A: Optimized exosome isolation protocol for cell culture supernatant and human plasma. J Extracell Vesicles 2015;4:27031.

-33 Grahame Hardie D: AMP-activated protein kinase: a key regulator of energy balance with many roles in human disease. J Intern Med 2014;276:543-559.

-34 Gwinn DM, Shackelford DB, Egan DF, Mihaylova MM, Mery A, Vasquez DS, Turk BE, Shaw RJ: AMPK phosphorylation of raptor mediates a metabolic checkpoint. Mol Cell 2008;30:214-226.

-35 Frost RA, Nystrom GJ, Lang CH: Endotoxin and interferon-gamma inhibit translation in skeletal muscle cells by stimulating nitric oxide synthase activity. Shock 2009;32:416-426.

-36 Crepaldi T, Bersani F, Scuoppo C, Accornero P, Prunotto C, Taulli R, Forni PE, Leo C, Chiarle R, Griffiths J, Glass DJ, Ponzetto C: Conditional activation of MET in differentiated skeletal muscle induces atrophy. J Biol Chem 2007;282:6812-6822.

-37 Miura T, Kishioka Y, Wakamatsu J, Hattori A, Hennebry A, Berry CJ, Sharma M, Kambadur R, Nishimura T: Decorin binds myostatin and modulates its activity to muscle cells. Biochem Biophys Res Commun 2006;340:675-680.

38 Zarfeshani A, Ngo S, Sheppard AM: Leucine alters hepatic glucose/lipid homeostasis via the myostatinAMP-activated protein kinase pathway - potential implications for nonalcoholic fatty liver disease. Clin Epigenetics 2014;6:27.

-39 Lundberg TR, Fernandez-Gonzalo R, Tesch PA: Exercise-induced AMPK activation does not interfere with muscle hypertrophy in response to resistance training in men. J Appl Physiol 2014;116:611-620.

40 Lee K, Ochi E, Song H, Nakazato K: Activation of AMP-activated protein kinase induce expression of Fox01, Fox03a, and myostatin after exercise-induced muscle damage. Biochem Biophys Res Commun 2015;466:289-294.

-41 Das AK, Yang QY, Fu X, Liang JF, Duarte MS, Zhu MJ, Trobridge GD, Du M: AMP-activated protein kinase stimulates myostatin expression in C2C12 cells. Biochem Biophys Res Commun 2012;427:36-40.

42 Li Y, Jiang B, Ensign WY, Vogt PK, Han J: Myogenic differentiation requires signalling through both phosphatidylinositol 3-kinase and p38 MAP kinase. Cell Signal 2000;12:751-757.

43 Meriane M, Charrasse S, Comunale F, Gauthier-Rouviere C: Transforming growth factor beta activates Rac1 and Cdc42Hs GTPases and the JNK pathway in skeletal muscle cells. Biol Cell 2002;94:535-543.

-44 Forterre A, Jalabert A, Berger E, Baudet M, Chikh K, Errazuriz E, De Larichaudy J, Chanon S, WeissGayet M, Hesse AM, Record M, Geloen A, Lefai E, Vidal H, Coute Y, Rome S: Proteomic analysis of C2C12 myoblast and myotube exosome-like vesicles: a new paradigm for myoblast-myotube cross talk? PLoS One 2014;9:e84153.

45 Clavel S, Siffroi-Fernandez S, Coldefy AS, Boulukos K, Pisani DF, Derijard B: Regulation of the intracellular localization of Foxo3a by stress-activated protein kinase signaling pathways in skeletal muscle cells. Mol Cell Biol 2010;30:470-480.

46 Doherty TJ: Invited review: Aging and sarcopenia. J Appl Physiol 2003;95:1717-1727. 


\section{Cellular Physiology Cell Physiol Biochem 2018;48:1829-1842 and Biochemistry Published online: Aungust 8, $2018 \quad \begin{aligned} & \text { DOI } 1018 \text { The Author(s). Published by S. Karger AG, Basel } \\ & \text { www.karger.com/cpb }\end{aligned}$ \\ Kim et al.: Effects of Exosomes on Muscle Differentiation in Inflamed Myotubes}

47 Matsuo Y, Gleitsmann K, Mangner N, Werner S, Fischer T, Bowen TS, Kricke A, Matsumoto Y, Kurabayashi M, Schuler G, Linke A, Adams V: Fibronectin type III domain containing 5 expression in skeletal muscle in chronic heart failure-relevance of inflammatory cytokines. J Cachexia Sarcopen 2015;6:62-72.

48 Serrano AL, Baeza-Raja B, Perdiguero E, Jardi M, Munoz-Canoves P: Interleukin-6 is an essential regulator of satellite cell-mediated skeletal muscle hypertrophy. Cell Metab 2008;7:33-44.

49 Hawke TJ, Garry DJ: Myogenic satellite cells: physiology to molecular biology. J Appl Physiol 2001;91:534551.

50 Ma JF, Sanchez BJ, Hall DT, Tremblay AK, Di Marco S, Gallouzi IE: STAT3 promotes IFNgamma/TNFalphainduced muscle wasting in an NF-kappaB-dependent and IL-6-independent manner. EMBO Mol Med 2017;9:622-637.

-51 Sente T, Van Berendoncks AM, Fransen E, Vrints CJ, Hoymans VY: Tumor necrosis factor-alpha impairs adiponectin signalling, mitochondrial biogenesis, and myogenesis in primary human myotubes cultures. Am J Physiol Heart Circul Physiol 2016;310:H1164-1175.

52 Trendelenburg AU, Meyer A, Rohner D, Boyle J, Hatakeyama S, Glass DJ: Myostatin reduces Akt/TORC1/ p70S6K signaling, inhibiting myoblast differentiation and myotube size. Am J Physiol Cell Physiol 2009;296:C1258-1270.

53 Salminen A, Hyttinen JM, Kaarniranta K: AMP-activated protein kinase inhibits NF-kappaB signaling and inflammation: impact on healthspan and lifespan. J Mol Med 2011;89:667-676.

-54 Williamson DL, Butler DC, Alway SE: AMPK inhibits myoblast differentiation through a PGC-1alphadependent mechanism. Am J Physiol Endocrinol Metab 2009;297:E304-314.

55 S EL Andalousssi, Mager I, Breakefield XO, Wood MJ: Extracellular vesicles: biology and emerging therapeutic opportunities. Nat Rev Drug Discov 2013;12:347-357.

-56 Aoi W, Sakuma K: Does regulation of skeletal muscle function involve circulating microRNAs? Front Physiol 2014;5:39.

-57 Aswad H, Forterre A, Wiklander OP, Vial G, Danty-Berger E, Jalabert A, Lamaziere A, Meugnier E, Pesenti S, Ott C, Chikh K, El-Andaloussi S, Vidal H, Lefai E, Rieusset J, Rome S: Exosomes participate in the alteration of muscle homeostasis during lipid-induced insulin resistance in mice. Diabetologia 2014;57:2155-2164.

58 Forterre A, Jalabert A, Berger E, Baudet M, Chikh K, Errazuriz E, De Larichaudy J, Chanon S, WeissGayet M, Hesse AM, Record M, Geloen A, Lefai E, Vidal H, Coute Y, Rome S: Proteomic analysis of C2C12 myoblast and myotube exosome-like vesicles: a new paradigm for myoblast-myotube cross talk? PLoS One 2014;9:e84153. 